SELECCIONES MATEMÁTICAS
Universidad Nacional de Trujillo
ISSN: $2411-1783$ (Online)
Vol. 03(02): $76-82(2016)$

\title{
Estabilidad de los Sistemas Lineales con Saltos Markovianos.
}

Stability of Linear Systems with Markovian Jumps.

Jorge Enrique Mayta Guillermo *

Received, Jun. 26, 2016

Accepted, Oct. 18, 2016.

DOI: http://dx.doi.org/10.17268/sel.mat.2016.02.03

\section{Resumen}

En este trabajo analizaremos la estabilidad de los sistemas lineales gobernados por una cadena de Markov, esta familia es conocida en la literatura especializada como sistemas lineales con saltos markovianos o por sus siglas en inglés MJLS como se denota en [1]. Los sistemas lineales gobernados por una cadena de Markov son sistemas dinámicos que presentan cambios abruptos. Damos algunas definiciones de estabilidad para el sistema MJLS, donde estos tipos de estabilidad son equivalentes siempre y cuando el espacio de estados de la cadena de Markov es finito.

Por último presentamos un teorema que caracteriza la estabilidad estocástica mediante una ecuación del tipo Lyapunov. El resultado que se presenta es una generalización de un teorema en la teoría clásica.

Palabras clave. sistemas lineales con saltos markovianos, estabilidad, cadenas de Markov.

\begin{abstract}
$n$ this work we will analyze the stability of linear systems governed by a Markov chain, this family is known in the specialized literature as linear systems with Markov jumps or by its acronyms in English MJLS as it is denoted in [1]. Linear systems governed by a Markov chain are dynamic systems with abrupt changes. We give some definitions of stability for the MJLS system, where these types of stability are equivalent as long as the state space of the Markov chain is finite. Finally we present a theorem that characterizes the stochastic stability by means of an equation of the Lyapunov type. The result is a generalization of a theorem in classical theory.
\end{abstract}

Keywords. Linear systems with Markov jumps, stability, Markov chains.

1. Introducción - Los sistemas lineales con saltos markovianos se ha venido utilizando desde la década de los 70 en diversas aéreas de investigación como por ejemplo este tipo de sistemas los podemos encontrar en sistemas de control aéreo [2], sistemas eléctricos [3],etc. La cadena de Markov asociada al sistema muda de estado aleatoriamente a medida que transcurre el tiempo. Cada estado de la cadena representa un modo de operar distinto del sistema. De esta manera, en lugar de un único sistema dinámico, se tienen en realidad muchos sistemas cambiando aleatoriamente y modelando todos ellos un mismo fenómeno. El modelo matemático resultante es un sistema dinámico estocástico conocido en la literatura como sistemas lineales con saltos markovianos o, por sus siglas en inglés, MJLS (ver p.ej. [1]).

1.1. Notaciones Básicas. En esta subsección se muestran las notaciones generales que se utiliza en todo el artículo son las siguientes. El espacio euclidiano de los números reales de $n$ dimensiones se denota por $\mathbb{R}^{n}$, el conjunto de números enteros no negativos, por $\mathbb{Z}_{+}$. El espacio vectorial de las matrices de orden

*Departamento de Matemáticas de la Pontificia Universidad Católica del Perú (jorge.maytag@pucp.pe).

This work is licensed under the Creative Commons Attribution-NoComercial-ShareAlike 4.0. 
$m \times n$ definida en $\mathbb{R}$ se representa por $\mathcal{M}_{m \times n}(\mathbb{R})$ y, para abreviar, $\mathcal{M}_{n}(\mathbb{R})$ denota el espacio de las matrices cuadradas de orden $n \times n$. La norma euclidiana en $\mathcal{M}_{n}(\mathbb{R})$ se denota por $\|$.$\| . La transposición y la traza$ de la matriz $A$ se denotan por $A^{T}$ y $\operatorname{tr}(A)$, respectivamente.

En vista que el fenómeno bajo estudio se torna aleatorio comenzamos el análisis introduciendo un espacio de probabilidad $(\Omega, \mathcal{F}, \operatorname{Pr})$, donde $\Omega$ es el espacio muestral, $\mathcal{F}$ es la $\sigma$-álgebra y $\operatorname{Pr}$ es la medida de probabilidad. El valor esperado se denotado por $E\{$.$\} . La función del indicador con respecto al evento$ $A \in \mathcal{F}$, se denota por $1_{A}$. El producto de Kronecker se denota con el símbolo $\otimes$ y el operador vec que transforma una matriz a un vector columna. La cadena de Markov se considera homogénea y que toma valores con espacio de estado

$$
\Sigma=\{1,2, \cdots, L\} .
$$

El vector de distribución de probabilidad inicial es denotado por $\pi$, donde $\pi=\left(\pi_{1}, \ldots, \pi_{L}\right), \pi_{i}=$ $\operatorname{Pr}(\theta(0)=i)$. La matriz de transición de probabilidades es denotada por $\left(p_{i j}\right)$, donde $p_{i j} \triangleq \operatorname{Pr}(\theta(k+1)=$ $j \mid \theta(k)=i)$. Por último el símbolo que denota la matriz identidad por $I$ cuya dimensión depende del contexto.

2. Sistemas Lineales con saltos markovianos. Consideremos el sistema

$$
x(k+1)=A_{\theta(k)} x(k)+B_{\theta(k)} u(k), x(0) \in \mathbb{R}^{n},
$$

donde para todo $i \in \Sigma, A_{i} \in \mathcal{M}_{n}(\mathbb{R}), B_{i} \in \mathcal{M}_{n \times p}(\mathbb{R}), x(k) \in \mathbb{R}^{n}, u(k) \in \mathbb{R}^{p}$. Vamos a asumir que las condiciones iniciales $x(0)$ y $\theta(0)$ son independientes, además $x(0)$ es de primer y segundo momento finito, es decir, $E\{\|x(0)\|\}<\infty$ y $E\left\{\|x(0)\|^{2}\right\}<\infty$ respectivamente.

Cuando $u(k)=0,(2.1)$ se transforma en el siguiente sistema no forzado:

$$
x(k+1)=A_{\theta(k)} x(k), x(0) \in \mathbb{R}^{n}
$$

Buena parte de los resultados de este artículo están referidos al sistema (2.2), también llamado en la literatura sistema homógeneo.

Definición 1. Se dice que el proceso estocástico $x(k)=\{x(k)\}_{k \in \mathbb{Z}_{+}}$es solución de (2.1) si para toda realización $\omega$ de $\theta(k)$, la ecuación (2.1) es satisfecha puntualmente, esto es,

$$
x(k+1, \omega ; x(0))=A_{\theta(k, \omega)} x(k, \omega ; 0)+B_{\theta(k, \omega)} u(k), \quad k \in \mathbb{Z}_{+} .
$$

A la solución $x(k)$ del sistema (2.1) se le llama también trayectoria solución.

Para presentar el siguiente resultado, tengamos en cuenta la notación siguiente:

$$
\operatorname{Pr}(\theta(k+1)=j \mid \theta(k))=p_{\theta(k) j},
$$

donde para cada $j$ fijo en $\Sigma, p_{\theta(k) j}$ es una variable aleatoria cuyos valores son $p_{i j}, i \in \Sigma$.

Lema 1. Sea $x(k)$ la trayectoria solución de (2.1) con $x(0)=x_{0}$. Entonces

$$
E\left\{1_{\{\theta(k+1)=j\}} \mid x(k), \theta(k)\right\}=p_{\theta(k) j}
$$

3. Las matrices $\mathcal{A}$ y $\mathcal{B}$. En esta sección se presentan las matrices $\mathcal{A}$ y $\mathcal{B}$ que serán de gran utilidad para analizar la estabilidad de (2.1). Por consiguiente, es de esperar que la estabilidad del sistema (2.2) pueda ser analizada a través del radio espectral de esta matriz. Para poder introducir estas matrices necesitamos previamente definir algunas matrices que están dadas en términos de la trayectoria solución del sistema. Estas matrices, que serán utilizadas a lo largo de este trabajo, son consistentes con aquellas definidas en la literatura (ver p.ej. [1]).

Para cada $k \in \mathbb{Z}_{+}, i \in \Sigma$, consideremos

$$
\begin{aligned}
Q(k) & \triangleq E\left\{x(k) x^{T}(k)\right\} \\
Q_{i}(k) \triangleq E\left\{x(k) x^{T}(k) 1_{\{\theta(k)=i\}}\right\} & \triangleq \operatorname{vec}\left(Q_{i}(k)\right) \\
q_{i}(k) & \\
q(k) & \triangleq\left(\begin{array}{c}
q_{1}(k) \\
\vdots \\
q_{L}(k)
\end{array}\right) \\
p_{i}(k) & \triangleq \operatorname{vec}\left(I_{n}\right) \pi_{i}(k)
\end{aligned}
$$




$$
p(k) \triangleq\left(\begin{array}{c}
p_{1}(k) \\
\vdots \\
p_{L}(k)
\end{array}\right)
$$

Observe que

$$
Q(k)=E\left\{\sum_{i=1}^{L} x(k) x^{T}(k) 1_{\{\theta(k)=i\}}\right\}=\sum_{i=1}^{L} Q_{i}(k)
$$

El lema 2 establece una ecuación recursiva para la matriz $Q_{i}(k)$, definida en (3.2). Esta ecuación es muy útil para la obtención de los principales resultados presentados en este trabajo (ver p.ej. Lema 3 )

Lema 2 ( [1]). Dado el sistema (2.2) con trayectoria solución $x(k)$, la matriz $Q_{j}(k)$ definida en (3.7) satisface la siguiente ecuación recursiva:

$$
Q_{j}(k+1)=\sum_{i=1}^{L} p_{i j} A_{i} Q_{i}(k) A_{i}^{T}, k \in \mathbb{Z}_{+}, j \in \Sigma
$$

A continuación se introducen las matrices $\mathcal{A}$ y $\mathcal{B}$ que serán de fundamental importancia para establecer diferentes resultados relacionados con la estabilidad de los sistemas (2.1) y (2.2).

$$
\begin{gathered}
\mathcal{A} \triangleq\left(\Pi^{T} \otimes I_{n^{2}}\right) \operatorname{diag}\left[A_{1} \otimes A_{1}, \ldots, A_{L} \otimes A_{L}\right] \\
\mathcal{B} \triangleq\left(\Pi^{T} \otimes I_{n^{2}}\right) \operatorname{diag}\left[B_{1} \otimes B_{1}, \ldots, B_{L} \otimes B_{L}\right]
\end{gathered}
$$

Note que estas matrices recogen la información de todos los parámetros del sistema y además guardan la información probabilística de la cadena de Markov. Enseguida se muestra que el sistema (2.2) puede ser transformado en una ecuación del tipo clásico mediante la matriz $\mathcal{A}$.

Lema 3 ( [1], [5]). Dado el sistema (2.2), el correspondiente vector columna $q(k)$, definido en (3.4), es una solución del sistema

$$
z(k+1)=\mathcal{A} z(k), z(0)=q(0) \in \mathbb{R}^{n^{2}}
$$

Para el caso no homogéneo, asumimos que la cadena de Markov es ergódica, de manera que para cualquier $\theta(0)$ existen $p_{i}$ (independiente de $\pi$ ) tal que $p_{i}=\lim _{k \rightarrow \infty} \pi_{i}(k)$. Además, la señal de entrada $u(k)$ es tomada como un proceso i.i.d. (variables aleatorias independientes e idénticamente distribuidas) con media cero, matriz de covarianza la matriz identidad $I$ e independiente de $\theta(k)$ y $x(0)$.

Tomando en cuenta las condiciones impuestas sobre $u(k)$ y siguiendo procedimientos análogos a los del lema anterior, se pueden establecer los dos siguientes resultados.

Lema 4. Dado sistema (2.1), el correspondiente vector columna $q(k)$, definido en (3.4), es una solución del sistema siguiente

$$
z(k+1)=\mathcal{A} z(k)+\mathcal{B} p(k), z(0)=q(0),
$$

donde $p(k)$ está definido en (3.6).

4. Estabilidad. A continuación presentamos algunas definiciones de estabilidad para el sistema (2.2) dadas en la literatura [6], [7].

Definición 2. Se dice que el sistema (2.2) es

a) Estable en media cuadrática $(E M C)$ si para cualquier $x(0) \in \mathbb{R}^{n}$ y cualquier $\theta(0)$ se tiene

$$
\lim _{k \rightarrow \infty} Q(k)=0
$$

donde $Q(k)$ está definido en (3.1).

b) Estocásticamente estable (EE) si para cualquier $x(0) \in \mathbb{R}^{n}$ y cualquier $\theta(0)$ se tiene

$$
E\left\{\sum_{k=0}^{\infty}\|x(k)\|^{2}\right\}<\infty
$$


c) Exponencialmente estable (EXE) si para cualquier $x(0) \in \mathbb{R}^{n}$ y cualquier $\theta(0)$ existen constantes $0<\alpha<1 \leq \beta$ tal que para todo $k \in \mathbb{Z}_{+}$se tiene

$$
E\left\{\|x(k)\|^{2}\right\} \leq \beta \alpha^{k} E\left\{\|x(0)\|^{2}\right\},
$$

donde $\alpha$ y $\beta$ son independientes de $x(0)$ y $\theta(0)$.

d) Segundo momento estable (SME) si para cualquier $x(0) \in \mathbb{R}^{n}$ y cualquier $\theta(0)$ se tiene

$$
\lim _{k \rightarrow+\infty} E\left\{\|x(k)\|^{2}\right\}=0
$$

e) Casi seguramente estable (CSE) si para cualquier $x(0) \in \mathbb{R}^{n}$ y cualquier $\theta(0)$ se tiene

$$
\operatorname{Pr}\left\{\lim _{k \rightarrow \infty}\|x(k)\|=0\right\}=1
$$

5. Estabilidad EMC y la matriz $\mathcal{A}$. En esta subsección analizamos la estabilidad de (2.1) en términos del radio espectral de la matriz $\mathcal{A}$.

5.1. Caso homogéneo. El lema 3 da una caracterización de la estabilidad EMC en términos de el vector $q(k)$ definido en (3.4).

Lema 5. El sistema (2.2) es EMC si y solo si para cualquier $x(0) \in \mathbb{R}^{n}$ y para cualquier $\theta(0)$ se cumple

$$
\lim _{k \rightarrow+\infty} q(k)=0 .
$$

El teorema 1 provee una herramienta de fácil implementación computacional para analizar si el sistema (2.2) es EMC mediante el radio espectral de la matriz $\mathcal{A}$.

Teorema 1. El sistema (2.2) es EMC si y solo si $\rho(\mathcal{A})<1$.

5.2. Caso no homogéneo. Comenzamos esta subsección definiendo la estabilidad en media cuadrática (EMC) para el sistema (2.1). Además, recordemos que en este caso consideramos que la cadena de Markov es ergódica, de manera que debido a (3.5) el límite $\lim _{k \rightarrow \infty} p_{i}(k)$ existe.

Definición 3. Se dice que el sistema (2.1) es estable en media cuadrática si para cualquier $x(0) \in \mathbb{R}^{n}$ y cualquier $\theta(0)$ existe una matriz simétrica $Q$ positivo semi-definida tal que

$$
\lim _{k \rightarrow \infty} Q(k)=Q \text {, }
$$

donde $Q(k)$ está definido en (3.1).

Lema 6 ( [?]). El sistema (2.1) es EMC si y solo si para cualquier condición inicial $x(0) \in \mathbb{R}^{n}$ y para cualquier $\theta(0)$ existen las matrices simétricas $Q_{i}$ positivo semi-definidas tal que

$$
Q_{i}=\lim _{k \rightarrow \infty} Q_{i}(k), i \in \Sigma
$$

Lema 7. El sistema (2.1) es EMC si y solo si para cualquier condición inicial $x(0) \in \mathbb{R}^{n}$ y para cualquier $\theta(0)$ existe un vector $q \in \mathbb{R}^{n^{2}}$ tal que

$$
q=\lim _{k \rightarrow \infty} q(k)
$$

donde $q(k)$ está definido en (3.4).

Teorema 2. El sistema (2.1) es EMC si y solo si $\rho(\mathcal{A})<1$

5.3. Ejemplos. En esta subsección se proporcionan diferentes ejemplos que ilustran los resultados presentados en las secciones previas. En particular se analiza la estabilidad del sistema estocástico en relación con la estabilidad de los subsistemas que lo conforman.

Ejemplo 1. En este ejemplo se muestra un sistema con todos sus modos estables, sin embargo, el sistema no es EMC.

$$
A_{1}=\left[\begin{array}{cc}
0 & 5 \\
0 & 0,2
\end{array}\right] \quad A_{2}=\left[\begin{array}{cc}
0,4 & 0 \\
10 & 0
\end{array}\right], \quad \Pi=\left[\begin{array}{cc}
0,9 & 0,1 \\
0,4 & 0,6
\end{array}\right]
$$


En este caso la matriz $\mathcal{A}$ es:

$$
\mathcal{A}=\left[\begin{array}{cccccccc}
0 & 0 & 0 & 22,5 & 0,064 & 0 & 0 & 0 \\
0 & 0 & 0 & 0,9 & 1,6 & 0 & 0 & 0 \\
0 & 0 & 0 & 0,9 & 1,6 & 0 & 0 & 0 \\
0 & 0 & 0 & 0,036 & 40 & 0 & 0 & 0 \\
0 & 0 & 0 & 2,5 & 0,096 & 0 & 0 & 0 \\
0 & 0 & 0 & 0,1 & 2,4 & 0 & 0 & 0 \\
0 & 0 & 0 & 0,1 & 2,4 & 0 & 0 & 0 \\
0 & 0 & 0 & 0,004 & 60 & 0 & 0 & 0
\end{array}\right] .
$$

Notamos que todos los modos son estables, pero como $\rho(\mathcal{A})=10,066>1$ el sistema no es EMC.

Ejemplo 2. En este ejemplo se muestra un sistema con todos sus modos inestables, sin embargo, el sistema es EMC.

$$
A_{1}=\left[\begin{array}{cc}
1,5 & -2 \\
0 & 0
\end{array}\right], \quad A_{2}=\left[\begin{array}{cc}
0 & 10 \\
0 & 2
\end{array}\right], \quad \Pi=\left[\begin{array}{cc}
0,2 & 0,8 \\
0,85 & 0,15
\end{array}\right]
$$

Claramente los modos del sistema son inestables. La matriz $\mathcal{A}$ es:

$$
\mathcal{A}=\left[\begin{array}{cccccccc}
0,45 & -0,6 & -0,6 & 0,8 & 0 & 0 & 0 & 85 \\
0 & 0 & 0 & 0 & 0 & 0 & 0 & 17 \\
0 & 0 & 0 & 0 & 0 & 0 & 0 & 17 \\
0 & 0 & 0 & 0 & 0 & 0 & 0 & 3,4 \\
1,8 & -2,4 & -2,4 & 3,2 & 0 & 0 & 0 & 15 \\
0 & 0 & 0 & 0 & 0 & 0 & 0 & 3 \\
0 & 0 & 0 & 0 & 0 & 0 & 0 & 3 \\
0 & 0 & 0 & 0 & 0 & 0 & 0 & 0,6
\end{array}\right]
$$

Como $\rho(\mathcal{A})=0,6<1$ el sistema es EMC.

Los ejemplos presentados permiten concluir que no hay relación entre la estabilidad de los modos y la estabilidad del sistema visto como un todo.

6. Estabilidad estocástica mediante la ecuación de Lyapunov. En esta sección se presenta una caracterización algebraica para la estabilidad estocástica del sistema (2.2) mediante un conjunto de ecuaciones de tipo Lyapunov. Observe que cuando el espacio de estados se reduce al conjunto unitario $\Sigma=\{1\}$ la ecuación (6.1) se convierte en la ecuación de Lyapunov para sistemas lineales sin saltos. La ecuación de Lyapunov que proponemos para el sistema (2.2) es de la forma siguiente:

$$
\sum_{j=1}^{L} p_{i j} A_{i}^{T} M_{j} A_{i}-M_{i}=-W_{i}, \quad i, j \in \Sigma,
$$

en esta ecuación todas las matrices están en el espacio $\mathcal{M}_{n}(\mathbb{R})$.

Teorema 3 ( [6]). El sistema (2.2) es EE si y solo si para cualquier conjunto de matrices simétricas $\left\{W_{i} ; i \in \Sigma\right\}$ positivo definidas existe un conjunto de matrices simétricas $\left\{M_{i} ; i \in \Sigma\right\}$ positivo definidas que satisfacen (6.1).

7. Relaciones entre las diferentes nociones de estabilidad. En esta sección se establecen la relaciones entre los diferentes tipos de estabilidad introducidos en la sección 4. Se prueba que bajo la condición de ser $\Sigma$ un espacio de estados finito, las nociones de estabilidad (a)-(d) son equivalentes y todas esta implican la estabilidad (e). Comencemos por el lema siguiente:

En el lema 8 se establecen dos desigualdades que van a ser útiles en la demostración de los teoremas 4 y 5 .

Lema 8. Sea $Q(k)$ la matriz definida en (3.1), entonces:

$$
\frac{1}{n} E\left\{\|x(k)\|^{2}\right\} \leq\|Q(k)\|_{\text {máx }} \leq E\left\{\|x(k)\|^{2}\right\}
$$


Teorema 4. El sistema (2.2) es EMC si y solo si es EE.

El siguiente resultado se basa fundamentalmente en las desigualdades (7.1).

Teorema 5. El sistema (2.2) es EE si y solo si es SME.

Para establecer la equivalencia entre la EMC y ES, teorema 6, será de utilidad la desigualdad (7.2).

Lema 9. Sea $A \in \mathcal{M}_{n}(\mathbb{R})$. Si $\rho(A)<1$ entonces existen $\beta \geq 1$ y $0<\gamma<1$ tal que

$$
\left\|A^{k}\right\|_{1} \leq \beta \gamma^{k}, \quad k \in \mathbb{Z}_{+}
$$

Estamos listos para presentar la equivalencia entre EMC y EXE.

Teorema 6. El sistema (2.2) es EMC si y solo si es EXE.

Teorema 7. Si el sistema (2.2) es EMC entonces es CSE.

Proof.

Del teorema anterior si el sistema es EMC entonces él es EXE, es decir, existen constantes $0<\alpha<$ $1 \leq \beta$ tal que para todo $k \in \mathbb{Z}_{+}$se tiene

$$
E\left\{\|x(k)\|^{2}\right\} \leq \beta \alpha^{k} E\left\{\|x(0)\|^{2}\right\},
$$

Esta desigualdad implica

$$
\sum_{k=0}^{\infty} E\left\{\|x(k)\|^{2}\right\} \leq \frac{\beta}{1-\alpha} E\left\{\|x(0)\|^{2}\right\}
$$

aplicando la desigualdad de Markov se sigue

$$
\operatorname{Pr}\{\|x(k)\| \geq \epsilon\} \leq \frac{E\left\{\|x(k)\|^{2}\right\}}{\epsilon^{2}}
$$

De (7.3) y (7.4) se obtiene

$$
\sum_{k=0}^{\infty} \operatorname{Pr}\{\|x(k)\| \geq \epsilon\} \leq \frac{1}{\epsilon^{2}} \sum_{k=0}^{\infty} E\left\{\|x(k)\|^{2}\right\} \leq \frac{\beta}{\epsilon^{2}(1-\alpha)} E\left\{\|x(0)\|^{2}\right\}
$$

Definiendo la sucesión de eventos

$$
A_{k}=\{\|x(k)\| \geq \epsilon\}
$$

se sigue de (7.5) y del lema de Borel-Cantelli que

$$
\operatorname{Pr}\left\{\bigcap_{k=1}^{\infty} \bigcup_{n=k}^{\infty} A_{n}\right\}=0
$$

Tomando complemento en esta igualdad se obtiene $\operatorname{Pr}\left\{\cup_{k=1}^{\infty} \cap_{n=k}^{\infty} A_{n}^{c}\right\}=1$, de donde $\operatorname{Pr}\left\{\lim _{n \rightarrow \infty}\|x(k)\|=0\right\}=$ 1. $\square$

Acknowledgments. Agradezco al Dr. Jorge Richard Chávez Fuentes por la orientación y elaboración de este artículo. De la misma manera agradezco a la Dirección General de Investigación de la Pontificia Universidad Católica del Perú (DGI-PUCP) por haber financiado gran parte de mis estudios de maestría a través de los proyectos DGI-2014-0019 y además un agradecimiento al comité cientifico de la IX-Fast Workshop on Applied and Computational Mathematics-2016 por la aceptación del trabajo.

[1] Costa, Oswaldo Luiz do Valle. Discrete-time Markov jump linear systems Springer, London, 2005.

[2] B. L. Stevens And F.L. Lewis Aircraft Modeling, Dynamics and Control, New York, NY: Wiley (1991).

[3] R.W. Newcomb The Semistate Description of Nonlinear Time-Variable Circuits, IEEE Trans. Autom. Control Vol CAS-28 No.1, January (1981) pp.62-71.

[4] YuAndong Ji. Chizeck Howard. Jump Linear Quadratic gaussian control: Steady-State Solution and Testable Conditions, Control-Theory and Advanced Technology Vol 6. No.3, (1990) pp.289-319. 
[5] TEJADA R. ARturo. Analysis of error recovery effects on digital flight control systems. Ms Dissertation, Old Dominion University 2002.

[6] Feng Xiangbo. Loparo Kenneth. \& Yuandong Ji. Chizeck Howard. Stochastic Stability properties of Jump Linear Systems,IEEE Transactions on Automatic Control, vol 37 No.1, 1992 pp. 38-53.

[7] FANG, Y. Stability analysis of linear control systems with uncertain parameters, Ph.D. Dissertation, Dept. of Systems, Control, and Industrial Engineering, Case Western Reserve University, Cleveland, OH.Springer, London, 1998.

[8] Mayta G. Jorge. Regularidad y Estabilidad de Sistemas Lineales con Saltos Markovianos en Tiempo Discreto. Tesis M.S, Dpto. Matemática, Pontificia Universidad Católica del Perú, Lima, Perú 2015. 